\title{
STABILITY OF ITERATIVE PROCEDURES FOR MULTIVALUED MAPS IN METRIC SPACES
}

\begin{abstract}
Let $(X, d)$ be a metric space and $T$ a self-map of $X$. Let $x_{n+1}=f\left(T, x_{n}\right)$ denote some iterative procedure. Let $\left\{x_{n}\right\}$ be convergent to a fixed point $u$ of $T$ and $\left\{y_{n}\right\}$ be an arbitrary sequence in $X$. Set $\varepsilon_{n}=d\left[y_{n+1}, f\left(T, y_{n}\right)\right], n=0,1,2, \ldots$, then the iterative procedure $f\left(T, x_{n}\right)$ is $T$-stable provided that $\lim _{n} \varepsilon_{n}=0$ implies that $\lim _{n} y_{n}=u$. This definition has been extended by Singh and Chadha [34] to discuss the problem of stability for multivalued operators on metric spaces. The purpose of this paper is to present a fixed point theorem for generalized multivalued contractions on a setting more general than metric spaces. The same is utilized to discuss the problem of stability of iterative procedures in multivalued analysis. Some special cases due to Stefan Czerwik and others are discussed as special cases.
\end{abstract}

\section{Introduction}

Let $(X, d)$ be a metric space and $T: X \rightarrow X$. Equations arising in many physical formulations can be expressed as a fixed point equation $T x=x$. Let $\left\{x_{n}\right\}$ be a sequence obtained by some iterative procedure $f\left(T, x_{n}\right)$ that converges to a fixed point of $T$. In practical computations, the sequence $\left\{x_{n}\right\}$ is calculated in the following manner. First we choose the initial approximation $x_{0} \in X$. Then we compute $x_{1}=f\left(T, x_{0}\right)$ but, due to rounding off or discretization of the function, we do not get the exact value of $x_{1}$, but a different value $y_{1}$ (say), which is close enough to $x_{1}$, i.e., $y_{1} \approx x_{1}$. Consequently, when computing $x_{2}=f\left(T, x_{1}\right)$ we actually compute $x_{2}$ as $x_{2}=f\left(T, y_{1}\right)$, and so, instead of the theoretical value $x_{2}$, we actually obtain $y_{2} \approx x_{2}$, and so on. In this way, instead of the actual sequence $\left\{x_{n}\right\}$ we obtain an approximate sequence $\left\{y_{n}\right\}$. The iterative procedure $f\left(T, x_{n}\right)$ is considered to be numerically stable if and only if the approximate sequence $\left\{y_{n}\right\}$ still converges to the desired solution of the equation $T x=x$. This kind of problem

\footnotetext{
Key words and phrases: Generalized contractions, multivalued maps, stability, fixed point, b-metric space.

2000 Mathematics Subject Classification: 47H10, 65J15, 41A25.
} 
for real-valued functions was first noticed by $M$. Urabe [37] in 1957. The study of stability of iterative procedures enjoys a celebrated place in applicable mathematics due to chaotic behavior of functions in discrete dynamics and other numerical computations using computer programs. For a detailed discussion on the role of stability of iterative procedures in mathematical sciences, especially in computational analysis, one may refer to Berinde [1], Chadha [2], Czerwik et. al. [10]-[11], Harder and Hicks [12]-[14], Ishikawa [15]-[16], Istratescu [17], Matkowski and Singh [20], Ortega and Rheinboldt [23], Osilike [24]-[26] and Rhoades [29]-[30]. We begin with the following classical theorem of stability on metric spaces due to A. M. Ostrowski [27].

THEOREM 1.1 (Ostrowski's stability theorem [27]). Let $(X, d)$ be a complete metric space and $T: X \rightarrow X$ a Banach contraction with contraction constant $k$, i.e. $d(T x, T y) \leq k d(x, y)$ for all $x, y \in X$, where $0 \leq k<1$. Let $u \in X$ be the fixed point of $T$. Let $x_{0} \in X$ and $x_{n+1}=T x_{n}, n=0,1,2, \ldots$ Suppose that $\left\{y_{n}\right\}$ is a sequence in $X$ and $\varepsilon_{n}=d\left(y_{n+1}, T y_{n}\right)$. Then

$$
d\left(u, y_{n+1}\right) \leq d\left(u, x_{n+1}\right)+k^{n+1} d\left(x_{0}, y_{0}\right)+\sum_{r=0}^{n} k^{n-r} \varepsilon_{r} .
$$

Moreover, $\lim _{n} y_{n}=u$ iff $\lim _{n} \epsilon_{n}=0$.

This result has been found very useful in numerical praxis and has been extended by several authors to various classes of single-valued operators (see Harder and Hicks [13]-[14], Jachymski [18], Liu [19], Osilike [24]-[26], Rhoades [29]-[30], Singh [34]-[35] and Zhou [38]). Inspired by the first result (Theorem 2.1 below ) on the stability of Picard iterative procedures for multivalued contractions on metric spaces due to Singh and Chadha [34], Czerwik, Dlutek and Singh [11] studied the stability of iterative procedures in b-metric spaces. In Section 4, we obtain a fixed point theorem for generalized multivalued contractions in b-metric spaces. Section 5 deals with the stability of iterative procedures for multivalued maps on a general setting.

\section{Stability for multivalued contractions}

Let $(X, d)$ be a metric space and $C L(X)$ the collection of all nonempty closed subsets of $X$. Then $(C L(X), H)$ stands for the well-known generalized Hausdorff metric space, where the generalized Hausdorff metric $H$ is induced by the metric $d$ (see, for instance, [22], [34] and [36]). A map $T: X \rightarrow$ $C L(X)$ is called a multivalued contraction iff there exists a positive number $q<1$ such that

$$
H(T x, T y) \leq q d(x, y)
$$

for all $x, y \in X$. Nadler, Jr. [21] (see also Rus et al. [32]) proved that 
a multivalued contraction on a complete space has a fixed point. Ciric [6] extended this result for generalized multivalued contractions on metric spaces. Singh and Chadha [34] extended Ostrowski's theorem to multivalued contractions using Nadler's theorem and introduced the following definition of stability of iterative procedures for multivalued maps. Let $X$ be a metric space and $T: X \rightarrow C L(X)$. Let $x_{0} \in X$ and $x_{n+1} \in f\left(T, x_{n}\right)$ denote the Picard iterative procedure for $T$. Let $\left\{x_{n}\right\}$ be convergent to a fixed point $u$ of $T$ and $\left\{y_{n}\right\}$ be an arbitrary sequence. Set $\varepsilon_{n}=H\left[y_{n+1}, f\left(T, y_{n}\right)\right], n=$ $0,1,2, \ldots$ The iterative procedure $f\left(T, x_{n}\right)$ is said to be $T$-stable provided that $\lim _{n} \varepsilon_{n}=0$ implies that $\lim _{n} y_{n}=u$. This definition is due to Hicks and Harder [13, 14] (see also [12]) when $T$ is a single-valued self-map of a metric space $X$. We cite here the first result on the stability of Picard iterative procedure for multivalued contractions.

THEOREM 2.1 ([34]). Let $X$ be a complete metric space and $T: X \rightarrow C L(X)$ satisfy the condition (1). Let $x_{0}$ be an arbitrary point in $X$ and $\left\{x_{n}\right\}$ an orbit for $T$ at $x_{0}$ such that $\left\{x_{n}\right\}$ is convergent to a fixed point $u$ of $T$. Let $\left\{y_{n}\right\}$ be a sequence in $X$, and set $\varepsilon_{n}=H\left(y_{n+1}, T y_{n}\right), n=0,1,2, \ldots$ Then

$$
d\left(u, y_{n+1}\right) \leq d\left(u, x_{n+1}\right)+q^{n+1} d\left(x_{0}, y_{0}\right)+\sum_{r=0}^{n} q^{n-r} \varepsilon_{r} .
$$

Further, if Tu is singleton then $\lim _{n} y_{n}=u$ iff $\lim _{n} \varepsilon_{n}=0$.

Czerwik, Dlutek and Singh [11] studied the stability of Picard iterative procedures for multivalued maps in b-metric spaces. In this paper, we obtain a fixed point theorem for generalized multivalued contractions in b-metric spaces and the same is used to study the stability of Picard iterative procedures for such maps.

\section{Preliminaries}

First we present some definitions and notations to be used in the sequel. Definition 3.1 (cf. [8]-[11]). Let $X$ be a set and $s \geq 1$ a given real number. A function $d: X \times X \rightarrow \mathbb{R}+$ is called a b-metric provided that for all $x, y, z \in X$,

$$
d(x, y)=0 \text { iff } x=y
$$

$$
d(x, y)=d(y, x)
$$

$$
d(x, z) \leq s[d(x, y)+d(y, z)] .
$$

The pair $(X, d)$ is called a b-metric space. In all that follows, $(X, d)$ is a b-metric space unless otherwise stated explicitly. As usual, the ordinary distance between a point $x \in X$ and a subset $A$ of $X$ will be denoted by $D(x, A)$. 
Notice that the class of b-metric spaces is effectively larger than that of metric spaces, since a b-metric space is a metric space when $s=1 \mathrm{in}(\mathrm{bm}-3)$. The following example due to Czerwik $[8,9]$ shows that a b-metric on $X$ need not be a metric on $X$.

EXAMPLE. Let $X=\left\{x_{1}, x_{2}, x_{3}\right\}$ and $d: X \times X \rightarrow \mathbb{R}+$ such that

$$
\begin{aligned}
& d\left(x_{1}, x_{2}\right)=a>2, d\left(x_{1}, x_{3}\right)=d\left(x_{2}, x_{3}\right)=1 \text { and } d\left(x_{n}, x_{n}\right)=0 \\
& d\left(x_{n}, x_{k}\right)=d\left(x_{k}, x_{n}\right), d\left(x_{n}, x_{k}\right) \leq \frac{a}{2}\left[d\left(x_{n}, x_{i}\right)+d\left(x_{i}, x_{k}\right)\right], n, k, i=1,2,3 .
\end{aligned}
$$

Then $(X, d)$ is a b-metric space.

Definition $3.2([9])$. Let $(X, d)$ be a b-metric space.The generalized Hausdorff b-metric $H$ on $C L(X)$, the collection of all non-empty closed subsets of $(X, d)$ is defined as follows:

$$
H(A, B):= \begin{cases}\max \left\{\sup _{x \in A} D(x, B), \sup _{y \in B} D(y, A)\right\} \\ & \text { if the maximum exists } \\ \infty, & \text { otherwise }\end{cases}
$$

where $A, B \in C L(X)$.

We record the following properties to be used frequently.

Lemma 3.1. For any $A, B, C \in C L(X)$,

(i) $D(x, B) \leq d(x, y)$, for any $y \in B$,

(ii) $D(A, B) \leq H(A, B)$

(iii) $H(A, C) \leq s[H(A, B)+H(B, C)]$ (cf. Czerwik [9]-[11]).

We shall need the following result as well.

LeMma 3.2 ([9]). Let $A$ and $B$ be distinct elements of $C L(X)$. Let $x \in A$. Then, for a given number $\alpha>1$, there exists a point $y$ in $B$ such that

$$
d(x, y) \leq \alpha H(A, B) \text {. }
$$

This result for a metric space is essentially due to Nadler, Jr. [21] and Ciric [6], (see also Rus [31]).

THEOREM 3.1 ([9]). If $(X, d)$ is a b-metric space, then the function $H$ : $C L(X) \times C L(X) \rightarrow[0, \infty)$ is a generalized Hausdorff b-metric in $C L(X)$. Moreover, if $(X, d)$ is complete, then $(C L(X), H)$ is also complete.

Definition 3.3. A map $T: X \rightarrow C L(X)$ is said to be a generalized multivalued contraction if and only if there exists a positive number $q<s^{-1}$ such that

(2) $H(T x, T y) \leq q \cdot \max \left\{d(x, y), D(x, T x), D(y, T y), \frac{1}{2}(D(x, T y)+D(y, T x))\right\}$ holds for all $x, y \in X$. 
An orbit of a multivalued map $T$ at a point $x_{0} \in X$ is a sequence $\left\{x_{n}\right.$ : $\left.x_{n} \in T x_{n-1}, n=1,2, \ldots\right\}$. A space $X$ is said to be $T$-orbitally complete iff every Cauchy sequence of the form $\left\{x_{n_{i}}: x_{n_{i}} \in T x_{n_{i-1}}\right\}$ converges in $X$. Evidently, if $X$ is a complete space then, $X$ is $T$-orbitally complete. These definitions along with Definition 3.3 are essentially due to Ciric $[6]$ in metric spaces.

\section{Fixed point theorems}

THEOREM 4.1. Let $X$ be a complete b-metric space and $T$ a generalized multivalued contraction on $X$. Then:

(i) for every $x_{0} \in X$ there exists an orbit $\left\{x_{n}\right\}$ of $T$ at $x_{0}$ and $u \in X$ such that $\lim _{n} x_{n}=u$;

(ii) the point $u$ is fixed under $T$, i.e, $u \in T u$.

Proof. If $s=1$ (cf. Def. 3.3) then all the conclusions follow from Ćiric [6]. So we need take $s>1$. Pick $x_{0} \in X$. Let $x_{1} \in T x_{0}$. We may assume that $H\left(T x_{0}, T x_{1}\right)>0$, since otherwise $T x_{0}=T x_{1}$, and $x_{1} \in T x_{1}$, i.e. $x_{1}$ is a fixed point of $T$. Let $\lambda$ be a real number such that $\lambda>0$ and $q^{1-\lambda}<1 / s$. Since $H\left(T x_{0}, T x_{1}\right)<q^{-\lambda} H\left(T x_{0}, T x_{1}\right)$ and $x_{1} \in T x_{0}$, by Lemma 3.2, there exists a point $x_{2} \in T x_{1}$ such that

$$
d\left(x_{1}, x_{2}\right) \leq q^{-\lambda} H\left(T x_{0}, T x_{1}\right) .
$$

Again assuming $T x_{1} \neq T x_{2}$ and selecting a point $x_{3}$ in $T x_{2}$, we have

$$
d\left(x_{2}, x_{3}\right) \leq q^{-\lambda} H\left(T x_{1}, T x_{2}\right) .
$$

Continuing the same way, we get a sequence $\left\{x_{n}: n \in \mathbb{N}\right\}$ of points of $X$ such that $x_{n+1} \in T x_{n}$ and

From (2),

$$
d\left(x_{n}, x_{n+1}\right) \leq q^{-\lambda} H\left(T x_{n-1}, T x_{n}\right), n=1,2, \ldots .
$$

$$
\begin{aligned}
& d\left(x_{n}, x_{n+1}\right) \leq q^{-\lambda} H\left(T x_{n-1}, T x_{n}\right) \\
& \leq q^{-\lambda} q \cdot \max \left\{d\left(x_{n-1}, x_{n}\right), D\left(x_{n-1}, T x_{n-1}\right), D\left(x_{n}, T x_{n}\right), \frac{1}{2} D\left(x_{n-1}, T x_{n}\right)\right\} \\
& \leq k \cdot \max \left\{d\left(x_{n-1}, x_{n}\right), d\left(x_{n-1}, x_{n}\right), d\left(x_{n}, x_{n+1}\right), \frac{1}{2} d\left(x_{n-1}, x_{n+1}\right)\right\},
\end{aligned}
$$

where $k=q^{1-\lambda}$.

Since $k<1$, the relation $d\left(x_{n}, x_{n+1}\right) \leq k d\left(x_{n}, x_{n+1}\right)$ implies $d\left(x_{n}, x_{n+1}\right)$ $=0$, we may assume that $x_{n} \neq x_{n+1}$ and

So

$$
d\left(x_{n}, x_{n+1}\right) \leq k \cdot \max \left\{d\left(x_{n-1}, x_{n}\right), \frac{1}{2} d\left(x_{n-1}, x_{n+1}\right)\right\} .
$$

$$
d\left(x_{n}, x_{n+1}\right) \leq k d\left(x_{n-1}, x_{n}\right), \text { or else }
$$




$$
d\left(x_{n}, x_{n+1}\right) \leq \frac{k s}{2}\left[d\left(x_{n-1}, x_{n}\right)+d\left(x_{n}, x_{n+1}\right)\right]
$$

This yields

that is

$$
d\left(x_{n}, x_{n+1}\right) \leq \max \left\{k, \frac{k s}{(2-k s)}\right\} d\left(x_{n-1}, x_{n}\right)
$$

$$
d\left(x_{n}, x_{n+1}\right) \leq k^{\prime} d\left(x_{n-1}, x_{n}\right), \quad \text { where } k^{\prime}=\max \left\{k, \frac{k s}{2-k s}\right\} .
$$

Repeat this argument to obtain

$$
d\left(x_{n}, x_{n+1}\right) \leq k^{\prime} d\left(x_{n-1}, x_{n}\right) \leq \cdots \leq k^{\prime n} d\left(x_{0}, x_{1}\right) .
$$

Since $k^{\prime}<1$, it follows that $\left\{x_{n}\right\}$ is a Cauchy sequence. Since $x_{n+1} \in T x_{n}$, $n=0,1,2, \ldots$, and $X$ is complete, there exists a point $u$ in $X$ such that $u=\lim _{n} x_{n}$. This proves (i).

By Lemma 3.1,

$$
\begin{aligned}
D(u, T u) \leq & H(u, T u) \leq s H\left(u, x_{n+1}\right)+s H\left(x_{n+1}, T u\right) \\
\leq & s d\left(u, x_{n+1}\right)+s H\left(T x_{n}, T u\right) \\
& \leq s d\left(u, x_{n+1}\right)+s q \cdot \max \left\{d\left(x_{n}, u\right), D\left(x_{n}, T x_{n}\right), D(u, T u),\right. \\
& \left.\frac{1}{2}\left[D\left(x_{n}, T u\right)+D\left(u, T x_{n}\right)\right]\right\} \\
& \leq s d\left(u, x_{n+1}\right)+s q \cdot \max \left\{d\left(x_{n}, u\right), d\left(x_{n}, x_{n+1}\right), D(u, T u),\right. \\
& \frac{1}{2}\left[D\left(x_{n}, T u\right)+d\left(u, x_{n+1}\right]\right\} .
\end{aligned}
$$

Making $n \rightarrow \infty$, this obtains $D(u, T u) \leq s q D(u, T u)$, and $u \in T u$.

If $T$ is a single-valued map in the above theorem then we obtain the following result.

THEOREM 4.2. Let $X$ be a complete b-metric space and $T: X \rightarrow X$ such that

(3) $d(T x, T y) \leq q \cdot \max \left\{d(x, y), d(x, T x), d(y, T y), \frac{1}{2}[d(x, T y)+d(y, T x)]\right\}$ for all $x, y \in X$ and $q<s^{-1}$. Then, $T$ has a unique fixed point $u$ such that $T u=u$.

Proof. It follows from Theorem 4.1 that $\mathrm{T}$ has a fixed point. The unicity of the fixed point follows usually.

We remark that Theorem 4.1 with $s=1$ is the main result of Ciric [6] and the condition (3) with $s=1$ is $\left(21^{\prime}\right)$ discussed and compared by Rhoades [28].

We close this section with the following open question:

Is Theorem 4.1 with (2) replaced by

$$
H(T x, T y) \leq q \cdot \max \{d(x, y), D(x, T x), D(y, T y), D(x, T y), D(y, T x)\}
$$
true? 
We remark that this question has an affirmative answer when $\mathrm{T}$ is a single-valued map on a complete metric space (cf. Ciric [7]).

\section{Stability results}

In the sequel we shall need the following

LEMMA 5.1 ([10], see also [11], [30]). Let $\left\{\varepsilon_{n}\right\}$ be a sequence of nonnegative real numbers. Let

$$
s_{n}=\sum_{r=0}^{n}(s \lambda)^{n-r} \varepsilon_{r} \text { where } 0 \leq s \lambda<1 .
$$

Then $\lim _{n} \varepsilon_{n}=0$ iff $\lim _{n} s_{n}=0$.

TheOREM 5.1. Let $(X, d)$ be a complete b-metric space and $T: X \rightarrow C L(X)$ satisfy (2). Let $x_{0} \in X$ and $\left\{x_{n}\right\}$ be an orbit for $T$ at $x_{0}$, i.e., $x_{n+1} \in T x_{n}$, $n=0,1,2 \ldots$, and $\left\{x_{n}\right\}$ converge to a fixed point $u$ of $T$. Let $\left\{y_{n}\right\}$ be $a$ sequence in $X$ and set $\varepsilon_{n}=H\left(y_{n+1}, T y_{n}\right), n=0,1,2, \ldots$ If $s^{2} q<1$ then

$$
\begin{aligned}
d\left(u, y_{n+1}\right) \leq & s d\left(u, x_{n+1}\right)+s(s \lambda)^{n+1} d\left(x_{0}, y_{0}\right) \\
& +s^{3} \lambda \sum_{r=0}^{n}(s \lambda)^{n-r} H_{r}+s^{2} \sum_{r=0}^{n}(s \lambda)^{n-r} \varepsilon_{r},
\end{aligned}
$$

where $\lambda=s q /\left(1-s^{2} q\right)$ and $H_{r}=H\left(x_{r}, T x_{r}\right)$. If $T u$ is singleton, then $\lim _{n} y_{n}=u$ iff $\lim _{n} \varepsilon_{n}=0$.

P r o of. From (2), for any $x, y \in X$, one of the following holds:

$$
\begin{aligned}
H(T x, T y) & \leq q d(x, y) \\
H(T x, T y) & \leq q D(x, T x) \leq q H(x, T x) \\
H(T x, T y) & \leq q D(y, T y) \leq q H(y, T y) \leq s q\{H(y, x)+H(x, T y)\} \\
& \leq s q d(x, y)+s q\{H(x, T x)+H(T x, T y)\}
\end{aligned}
$$

i.e, $H(T x, T y) \leq \lambda\{d(x, y)+s H(x, T x)\}$;

$$
\begin{aligned}
H(T x, T y) & \leq \frac{q}{2}\{D(x, T y)+D(y, T x)\} \leq \frac{q}{2}\{H(x, T y)+H(y, T x)\} \\
& \leq \frac{s q}{2}\{H(x, T x)+H(T x, T y)+H(y, x)+H(x, T x)\}
\end{aligned}
$$

i.e, $H(T x, T y) \leq s q /(2-s q)\{2 H(x, T x)+d(y, x)\}$. Therefore, in all the cases,

$$
H(T x, T y) \leq \lambda d(x, y)+s \lambda H(x, T x) .
$$

We notice that, in view of Theorem 4.1, the conditions of Theorem 5.1 guarantee that $\mathrm{T}$ has a fixed point.

For any nonnegative integer $n$,

$$
d\left(x_{n+1}, y_{n+1}\right) \leq H\left(T x_{n}, y_{n+1}\right) \leq s\left[H\left(T x_{n}, T y_{n}\right)+H\left(T y_{n}, y_{n+1}\right]\right.
$$


Then, by virtue of (5), we obtain

$$
\begin{aligned}
d\left(x_{n+1}, y_{n+1}\right) \leq & s \lambda d\left(x_{n}, y_{n}\right)+s^{2} \lambda H\left(x_{n}, T x_{n}\right)+s \varepsilon_{n} \\
\leq & s^{2} \lambda\left[\lambda d\left(x_{n-1}, y_{n-1}\right)+s \lambda H\left(x_{n-1}, T x_{n-1}\right)+\varepsilon_{n-1}\right] \\
& +s^{2} \lambda H_{n}+s \varepsilon_{n} \\
\leq & (s \lambda)^{2} d\left(x_{n-1}, y_{n-1} 1\right)+s(s \lambda)^{2} H_{n-1}+s(s \lambda) H_{n} \\
& +s\left(s \lambda \varepsilon_{n-1}+\varepsilon_{n}\right) .
\end{aligned}
$$

Repeating this process $(n-1)$ times, we get

$$
\begin{aligned}
d\left(x_{n+1}, y_{n+1}\right) \leq & (s \lambda)^{n+1} d\left(x_{0}, y_{0}\right)+s^{2} \lambda \sum_{r=0}^{n}(s \lambda)^{n-r} H_{r} \\
& +s \sum_{r=0}^{n}(s \lambda)^{n-r} \varepsilon_{r} .
\end{aligned}
$$

Consequently,

$$
d\left(u, y_{n+1}\right) \leq s d\left(u, x_{n+1}\right)+s d\left(x_{n+1}, y_{n+1}\right)
$$

yields the inequality (4).

Assume that $\lim _{n} y_{n}=u$. Then by (5),

$$
\begin{aligned}
\varepsilon_{n} & =H\left(y_{n+1}, T y_{n}\right) \leq s H\left(y_{n+1}, u\right)+s H\left(u, T y_{n}\right) \\
& \leq s d\left(y_{n+1}, u\right)+s H\left(T u, T y_{n}\right) \\
& \leq s d\left(y_{n+1}, u\right)+s \lambda d\left(u, y_{n}\right)+s^{2} \lambda H(u, T u) \rightarrow 0 \text { as } n \rightarrow \infty .
\end{aligned}
$$

Suppose $\lim _{n} \varepsilon_{n}=0$. Since $0 \leq s \lambda<1$ and $\lim _{n} x_{n}=u$, first two terms on the right hand side of (4) vanish in the limit. Applying Lemma 5.1 to the last term of inequality (4), we see that $s^{2} \lim _{n} \sum_{r=0}^{n}(s \lambda)^{n-r} \varepsilon_{r}=0$.

Finally we show that, $s^{3} \lambda \lim _{n} \sum_{\tau=0}^{n}(s \lambda)^{n-r} H_{r}=0$.

Let $A$ denote the lower triangular matrix with entries $a_{n r}=(s \lambda)^{n-r}$. Then, $\lim _{n} a_{n r}=0$ for each $r$, and

$$
\lim _{n}\left(\sum_{r=0}^{n}\left(a_{n r}\right)\right)=\lim _{n}\left(\frac{1-(s \lambda)^{n+1}}{1-s \lambda}\right)=\frac{1}{1-s \lambda} .
$$

Therefore, $A$ is multiplicative, i.e, for any convergent sequence $\left\{s_{n}\right\}$, $\lim _{n} A\left(s_{n}\right)=\frac{1}{1-s \lambda} \lim _{n} s_{n}$. Thus, $s^{3} \lambda \lim _{n} \sum_{r=0}^{n}(s \lambda)^{n-r} H_{r}=0$ (cf. [30], p. 692).

REMARK 5.1. If $s=1$ and $T$ is single-valued, then the following result is obtained from Theorem 5.1.

Corollary 5.1. Let $X$ be a complete metric space and $T: X \rightarrow X$ such that 
(6) $d(T x, T y) \leq q \cdot \max \left\{d(x, y), d(x, T x), d(y, T y), \frac{1}{2}(d(x, T y)+d(y, T x))\right\}$ holds for all $x, y \in X$. Let $u$ be the fixed point of $T$. Let $x_{0} \in X, x_{n+1}=$ $T x_{n},\left\{y_{n}\right\} \subset X$ and set $\varepsilon_{n}=d\left(y_{n+1}, T y_{n}\right), n=0,1,2, \ldots$ If $2 q<1$ then $d\left(u, y_{n+1}\right) \leq d\left(u, x_{n+1}\right)+q^{n+1} d\left(x_{0}, y_{0}\right)+m q \sum_{r=0}^{n} q^{n-r} d\left(x_{r}, x_{r+1}\right)+\sum_{r=0}^{n} q^{n-r} \varepsilon_{r}$, where $m=(1-q)^{-1}$. Then $\lim _{n} y_{n}=u$ iff $\lim _{n} \varepsilon_{n},=0$.

We remark that the condition (6) is a slightly different from the following condition studied by Rhoades [30].

$$
d(T x, T y) \leq c . m a x\left\{d(x, y), \frac{1}{2}(d(x, T x)+d(y, T y)), d(x, T y), d(y, T x)\right\}
$$

where $0 \leq c<1$. Indeed, Rhoades [op. cit.] has shown that the Picard iterative procedure for such a map $T$ on a complete metric space is stable.

THEOREM 5.2. Let the hypotheses of Theorem 5.1 hold, wherein $\varepsilon_{n}$ is replaced by $\varepsilon_{n}=d\left(y_{n+1}, u_{n}\right), u_{n} \in T y_{n}, n=0,1,2, \ldots ;$ and $\lambda<s^{-2}$. Then

$$
\begin{aligned}
d\left(u, y_{n+1}\right) \leq & s d\left(u, x_{n+1}\right)+s g^{n+1} d\left(x_{0}, y_{0}\right)+s^{2} \sum_{r=0}^{n} g^{n+1-r} H_{r} \\
& +s^{2} \sum_{r=0}^{n} g^{n-r}\left(s h_{r}+\varepsilon_{r}\right),
\end{aligned}
$$

where $g=s^{2} \lambda$ and $h_{r}=H\left(x_{r+1}, T x_{r}\right)$. Moreover, if $T u$ is singleton, then

$$
\lim _{n} y_{n}=u \text { iff } \lim _{n} \varepsilon_{n}=0 \text {. }
$$

Proof. First we show the following

$$
\begin{aligned}
d\left(x_{n+1}, y_{n+1}\right) \leq & g^{n+1} d\left(x_{0}, y_{0}\right)+s \sum_{r=0}^{n} g^{n+1-r} H_{r} \\
& +s \sum_{r=0}^{n} g^{n-r}\left(s h_{r}+\varepsilon_{r}\right), \quad n=0,1,2, \ldots
\end{aligned}
$$

We use (5) to have

$$
\begin{aligned}
d\left(x_{1}, y_{1}\right) & \leq s\left[d\left(x_{1}, u_{0}\right)+d\left(u_{0}, y_{1}\right)\right] \leq s H\left(x_{1}, T y_{0}\right)+s \varepsilon_{0} \\
& \leq s\left[s H\left(x_{1}, T x_{0}\right)+s H\left(T x_{0}, T y_{0}\right)\right]+s \varepsilon_{0}
\end{aligned}
$$

that is,

$$
d\left(x_{1}, y_{1}\right) \leq s^{2} \lambda d\left(x_{0}, y_{0}\right)+s\left(s^{2} \lambda\right) H_{0}+s\left(s h_{0}+\varepsilon_{0}\right) .
$$

This is the inequality (8) (for $n=0$ ). 
Now one may prove the inequality ( 8 ) by induction. Consequently, the relation (7) follows directly from

$$
d\left(u, y_{n+1}\right) \leq s d\left(u, x_{n+1}\right)+s d\left(x_{n+1}, y_{n+1}\right) .
$$

Assume $\lim _{n} y_{n}=u$. Then $\varepsilon_{n}=d\left(y_{n+1}, u_{n}\right) \leq H\left(y_{n+1}, T y_{n}\right)$ and, as in Theorem 5.1, $\lim _{n} \varepsilon_{n}=0$. Suppose $\lim _{n} \varepsilon_{n}=0$. Then from (5),

$$
\begin{aligned}
h_{r} & =H\left(x_{r+1}, T x_{r}\right) \leq s H\left(x_{r+1}, T u\right)+s H\left(T u, T x_{r}\right) \\
& \leq s d\left(x_{r+1}, u\right)+s \lambda d\left(u, x_{r}\right)+s^{2} \lambda H(u, T u) \rightarrow 0 \text { as } r \rightarrow \infty .
\end{aligned}
$$

Now, since $0 \leq g<1$, in view of the proof of Theorem 5.1, $\lim _{n} y_{n}=u$. This completes the proof.

REMARK 5.2. Theorems 5.1 and 5.2 are extensions of the corresponding results of Czerwik, Dlutek and Singh [11].

REMARK 5.3. For $s=1$, Theorems 5.1 and 5.2 include the similar results of Singh and Chadha [34].

REMARK 5.4. If we set $s=1$ in Theorems 5.1 and 5.2 , we obtain the similar results of Singh and Bhatnagar [33]. There is a scope to study stability problem for results presented in [4], [5], [15] and [16] on the setting of $b$-metric spaces.

Acknowledgement. The first author thanks the editors for their patience.

\section{References}

[1] V. Berinde, Iterative Approximation of Fixed Points, Efemeride Publishing House, Baia Mare, 2002.

[2] V. Chadha, A study in fixed point theory and stability problems, $\mathrm{Ph} . \mathrm{D}$.Thesis, Gurukula Kangri Univ., Hardwar, India, 1994.

[3] C. E. Chidume and C. Moore, The solution by iteration of nonlinear equations in uniformly smooth Banach spaces, J. Math. Anal. Appl. 215 (1997), 132-146.

[4] C. E. Chidume and C. Moore, Fixed point iterations for pseudo contractive maps, Proc. Amer. Math. Soc. 127 (1999), 1163-1170.

[5] C. E. Chid ume and M. O. Osilike, Fixed point iterations for quasi-contractive maps in uniformly smooth Banach spaces, Bull. Korean Math. Soc. 30 (1993), 201-212.

[6] Lj. B. Ciric, Fixed points for generalized multi-valued contractions, Mat. Vesnik 9 (24) (1972), 265-272.

[7] Lj. B. Ciric, A generalization of Banach's contraction principle, Proc. Amer. Math Soc. 45 (1974), 267-273.

[8] S. Czerwik, Contraction mappings in b-metric spaces, Acta Math. Inform. Univ. Ostraveiensis 1 (1993), 5-11. 
[9] S. Czerwik, Nonlinear set-valued contraction mappings in b-metric spaces, Atti. Sem. Mat. Fis. Univ. Modena, XLVI (1998), 263-276.

[10] S. Czerwik, K. Dlutek, and S. L. Singh, Round-off stability of iteration procedures for operators in b-metric spaces, J. Natur. Phys. Sci. 11 (1997), 87-94.

[11] S. Czerwik, K. Dlutek, and S. L. Singh, Round-off stability of iteration procedures for set-valued operators in b-metric spaces, J. Natur. Phys. Sci. 15 (1-2) (2001), 1-8.

[12] A. M. Harder, Fixed points theory and stability results for fuxed point iteration procedures, Ph.D. Thesis, Univ. Missouri-Rolla, 1987.

[13] A. M. Harder and T. L. Hicks, A stable iteration procedure for non-expansive mappings, Math. Japon. 33 (1988), 687-692.

[14] A. M. Harder and T. L. Hicks, Stability results for fixed point iteration procedures, Math. Japon. 33 (1988), 693-706.

[15] S. Ishikawa, Fixed points by a new iteration method, Proc. Amer. Math. Soc. 44 (1974), 147-150.

[16] S. Ishikawa, Fixed points and iteration of a non expansive mapping in a Banach space, Proc. Amer. Math. Soc. 59 (1976), 65-71.

[17] V. I. Istratescu, Fixed Point Theory, D. Reidel Publishing Co., Dordrecht; Holland, 1981.

[18] J. R. Jachymski, An extension of A. Ostrowski's theorem on the round-off stability of iterations, Aequations Math. 53 (1997), 242-253.

[19] L. S. Liu, Ishikawa and Mann iterative process with errors for nonlinear strongly accretive mappings in Banach spaces, J. Math. Anal. Appl. 194 (1995), 114-125.

[20] J. Matkowski and S. L. Singh, Round-off stability of functional iterations on product spaces, Indian J. Math. 39 (3) (1997), 275-286.

[21] S. B. Nadler, Jr., Multivalued contraction mappings, Pacific J. Math. 30 (1969), $475-488$.

[22] S. B. Nadler, Jr., Hyperspaces of Sets, Marcel-Dekker, New York, 1978.

[23] J. M. Ortega and W. C. Rheinboldt, Iterative Solutions of Nonlinear Equations in Several Variables, Academic Press, New York/London, 1970.

[24] M. O. Osilike, Stability results for the Ishikawa fixed point iteration procedure, Indian J. Pure Appl. Math. 26 (1995), 937-945.

[25] M. O. Osilike, Iterative solution of nonlinear equations of f-strongly accretive type, J. Math. Anal. Appl. 200 (1996), 259-271.

[26] M. O. Osilike and A. Udomene, Short proofs of stability results for fixed point iteration procedures for a class of contractive type mappings, Indian J. Pure Appl. Math. 30 (12) (1999), 1229-1234.

[27] A. M. Ostrowski, The round-off stability of iterations, Z. Angew. Math. Mech. 47 (1967), 77-81.

[28] B. E. Rhoades, A comparison of various definitions of contractive mappings, Trans. Amer. Math. Soc. 226 (1977), 257-290.

[29] B. E. Rhoades, Fixed point theorems and stability results for fixed point iteration procedures, Indian J. Pure Appl. Math. 21 (1990), 1-9.

[30] B. E. Rhoades, Fixed point theorems and stability results for fixed point iteration procedures-II, Indian J. Pure Appl. Math. 24 (1993), 691-703.

[31] I. A. Rus, Fixed point theorems for multivalued mappings in complete metric spaces, Math. Japon. 20 (1975), 21-24.

[32] I. A. Rus, A. Petrusel and G. Petrusel, Fixed point theory: 1950-2000: Romanian Contributions, House of the Book of Science, Cluj-Napoca, 2002. 
[33] S. L. Singh and C. Bhatnagar, Stability of iterative procedures for multivalued operators, Proc. of the Conference on Recent Trends in Mathematics and its Applications, 28th Frb.- 1st March, 2003, Indian Society of Math. \& Mat. Sci., Gorakhpur, 2003, pp. 25-34.

[34] S. L. Singh and V. Chadha, Round-off stability of iterations for multivalued operators, C. R. Math. Rep. Acad. Sci. Canada 17 (5) (1995), 187-192.

[35] S. L. Singh, S. N. Mishra and V. Chadha, Round-off stability of iterations on product spaces, C. R. Math. Rep. Acad. Sci. Canada (1994).

[36] S. L. Singh and J. H. M. Whitfield, Contractors and fixed points, Colloq. Math. 25 (1988), 279-288.

[37] M. Urabe, Convergence of numerical iteration in solution of equation, J. Sci. Hiroshima Univ. Sér A. 19 (1956), 479-489.

[38] H. Y. Zhou, Stable iteration procedures for strong pseudocontractions and nonlinear equations involving accretive operators without Lipschitz assumption, J. Math. Anal. Appl. 230 (1999), 1-10.

S. L. Singh and Charu Bhatnagar GURUKULA KANGRI VISHWAVIDYALAYA, HARDWAR

(SLS) 21, GOVIND NAGAR, RISHIKESH - 249201, INDIA

(CB) N-112, SHIVALIK NAGAR, B.H.E.L., HARDWAR-249403, INDIA

e-mail: vedicmri@sancharnet.in

S. N. Mishra

DEPARTMENT OF MATHEMATICS

UNIVERSITY OF TRANSKEI

UMTATA 5117, SOUTH AFRICA

e-mail: mishra@getafix.utr.ac.za

Received March 20, 2004. 